\title{
不规则格点上的车贝雪夫多项式展开问题
}

\author{
周 家 斌 \\ (中国科学院大气物理研究所)
}

在 $x$ 轴的某区间上取 $I_{0}$ 个格点, 且令其格点值为 $1,2, \cdots I_{0}$, 则这些等距格点上的车贝雪. 夫 (Чебышев) 多项式定义为:

$$
\left\{\begin{array}{l}
\varphi_{0}(x)=1, \\
\varphi_{1}(x)=x-\frac{I_{0}-1}{2}, \\
\varphi_{1}(x)=\varphi_{1}^{2}(x)-\frac{I_{0}^{2}-1}{12}, \\
\cdots \cdots \cdots \cdots \\
\left(x=1,2, \cdots I_{0}\right)
\end{array}\right.
$$

递推公式为

$$
\varphi_{k+1}(x)=\varphi_{1}(x) \varphi_{k}(x)-\frac{k^{2}\left(I_{0}^{2}-k^{2}\right)}{4\left(4 k^{2}-1\right)} \varphi_{k-1}(x) .
$$

车贝雪夫多项式是正交的, 满足

$$
\sum_{x=1}^{I_{0}} \varphi_{k}(x) \varphi_{s}(x)=0(k \neq s) .
$$

定义在同一区间上的函数 $z(x)$ 可用车贝雪夫多项式展开, 即

$$
z(x)=\sum_{k=0}^{K_{0}} A_{k} \varphi_{k}(x),
$$

其中 $K_{0}$ 为展开用多项式最高阶数，而

展开的误差可用下式估计

$$
\begin{gathered}
A_{k}=\sum_{x=1}^{I_{0}} z(x) \varphi_{k}(x) / \sum_{x=1}^{I_{0}} \varphi_{k}^{2}(x), \\
\left(k=0,1,2, \cdots K_{0}\right) .
\end{gathered}
$$

在二维情况下, 与(4)、(5)、(6)式相应的公式为

$$
\sum_{K_{0}}^{2}=\sum_{x=1}^{I_{0}} z^{2}(x)-\sum_{k=0}^{R_{0}}\left[A_{k}^{2} \sum_{x=1}^{I_{0}} \varphi_{k}^{2}(x)\right] .
$$

$$
z\left(x_{1}, x_{2}\right)=\sum_{k=0}^{K_{0}} \sum_{s=0}^{s_{0}} A_{k s} \varphi_{k}\left(x_{1}\right) \psi_{s}\left(x_{2}\right),
$$

本文 1980 年 9 月 29 日收到. 


$$
\begin{gathered}
A_{k s}=\sum_{x_{1}=1}^{I_{0}} \sum_{x_{n}=3}^{J_{0}} z\left(x_{1}, x_{2}\right) \varphi_{k}\left(x_{1}\right) \psi_{s}\left(x_{2}\right) / \sum_{x_{1}=1}^{I_{0}} \sum_{x_{1}=1}^{J_{0}} \varphi_{k}^{2}\left(x_{1}\right) \psi_{s}^{2}\left(x_{2}\right), \\
\left(k=0,1,2, \cdots K_{0} ; s=0,1,2, \cdots S_{0}\right) \\
\sum_{K_{0} \cdot S_{n}}^{2}=\sum_{x_{1}=1}^{I_{0}} \sum_{x_{n}=1}^{J_{0}} z^{2}\left(x_{1}, x_{2}\right)-\sum_{k=0}^{K_{0}} \sum_{s=0}^{s_{0}}\left[A_{k s}^{2} \sum_{x_{1}=1}^{I_{0}} \sum_{x_{2}=1}^{J_{0}} \varphi_{k}^{2}\left(x_{1}\right) \psi_{s}^{2}\left(x_{2}\right)\right] .
\end{gathered}
$$

\section{以上各式的推导见文献 [1].}

上述车贝雪夫多项式的有关公式都只与 $x_{1}=1,2, \cdots I_{0} ; x_{2}=1,2, \cdots J_{0}$ 这种特殊的 离散值有关. 利用这一点, 我们可以把车贝雪夫多项式推广到不规则格点上.

考虑 $x$ 轴上的不规则格点 $x=x_{1}, x_{2}, \cdots x_{I_{0}}$.

引进一个非线性变换

$$
\xi=F X,
$$

而使 $\xi=1,2, \cdots, I_{0}$. 这只要将 $\xi$ 取成 $x_{i}$ 的序号就可以了. 此时, $\xi$ 是等距格点, 我们就 可以应用公式 (1)-(6), 而只要将其中的 $x$ 理解为 $\xi$ 就行了.

对于二维情况下的不规则格点 $\left(x_{1 i}, x_{2 i}\right)\left(i=1,2, \cdots I_{0} ; j=1,2, \cdots J_{0}\right)$, 同样可以引 进非线性变换

$$
\Xi=F x
$$

其中

$$
\Xi=\left(\xi_{1}, \xi_{2}\right), X=\left(x_{1}, x_{2}\right),
$$

要使 $\left(\xi_{1}, \xi_{2}\right)$ 成为等距的, 这只要将其取成 $\left(x_{1 i}, x_{2 i}\right)$ 的二维序号 $(1,1),(2,1), \cdots\left(I_{0}, 1\right)$, $(1,2),(2,2), \cdots\left(I_{0}, 2\right), \cdots\left(1, J_{0}\right),\left(2, J_{0}\right), \cdots\left(I_{0}, J_{0}\right)$ 就可以了.

三维及其以上的情况类似,不难推广. 这只要将相应的格点 $\left(x_{1 i}, x_{2 i}, \cdots x_{n t}\right)$ 变换成多维 序号 $\left(\xi_{1 i}, \xi_{2 i}, \cdots \xi_{n t}\right)$ 就行了.

下面举出两个例子说明上述推广的应用.

例 1 . 风速垂直分布的展开.

垂直方向取 18 层资料，即 $x=0.5,1,1.5,2,3,4,5,6,7,8,9,10,12,14,16,18,20$ ， 22 公里. 用序号 $\xi_{i}=1,2, \cdots 18$ 代替自变量 $x_{i}$, 则可以在垂直方向的 18 个不规则格点上 把风速资料展开. 用三个站 90 天资料进行计算, 结果说明只要取 7-12 个系数就可以表达 18 层资料, 其误差不超过 2 米/秒.

例 2. 北太平洋月平均海面温度分布的展开。

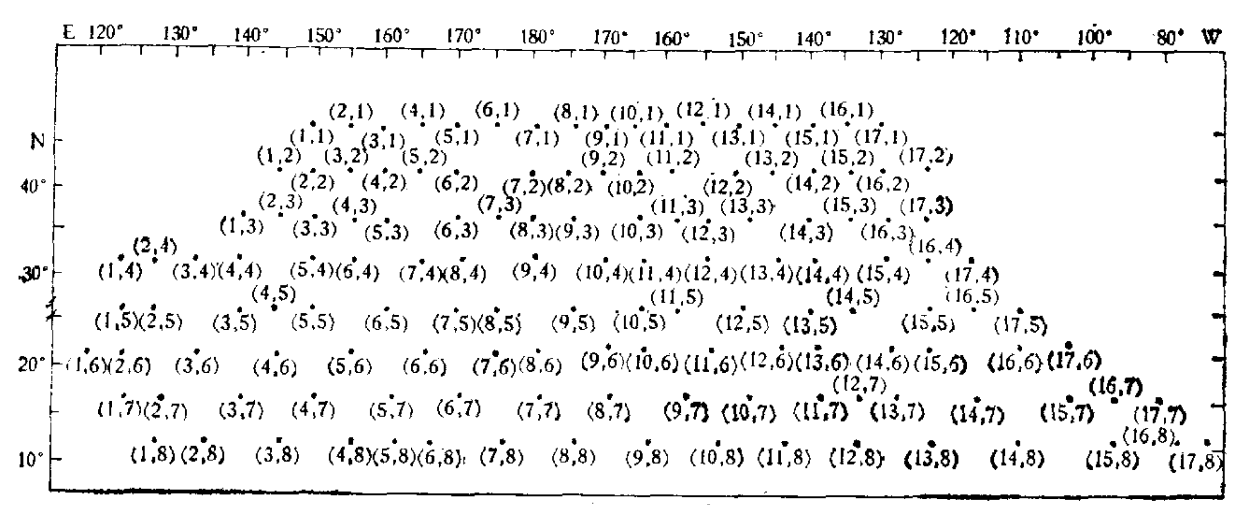

图 1 海温格点分布与序号的关系 
图 1 给出海温的格点分布及相应的序号.

根据统计, 要达到 0.3 度的准确度,所需系数为格点总数的 $20-30 \%$.

推广后的车贝雪夫多项式具有两个显著的优点。其一, 每个多项式都有标准图形。其二, 可以用于不规则格点. 而一般气象上常用的正交函数如三角函数、球函数、自然正交函数都不 "能同时具备这两个优点.

用车贝雪夫多项式将历史资料 (例如天气图) 展开, 研究其系数的历史演变, 可以分析天 气过程. 分析不同天气图的系数特征, 可以建立相应的环流分型方案和识别相似天气图. 将 预报因子和预报量取成气象场的展开式系数，我们就可以用面上的资料来预报气象要素的空 间分布, 从而使目前的单点统计预报推进到面的预报。在存储和传递资料时, 若将其用车贝雪 夫多项式展开, 则可以将原始资料的传递和存贮用系数的相应处理代替, 从而提高资料传递和 存贮的效率. 另外,车贝雪夫多项式还可以用于气象场的客观分析.

致谢: 在本文写作过程中,作者与系统科学研究所罗霄林同志进行过有益的讨论. 大气物理研究所李欣. 荣同志参加了本文部分计算工作,李荣凤同志为本文提供了风速资料．作者谨致谢意.

\section{参考 文献}

[1] Багров Н. А., Труды ЦИП, 64 (1958), 3-25. 